\title{
Uso del mercadeo estratégico en las Mipymes de Bogotá
}

\author{
Fecha de recepción: 24 de marzo de 2011 - Aprobación: 11 de noviembre de 2011
}

Sandra Patricia Rojas Berrio - Ricardo Arturo Vega Rodríguez

\begin{abstract}
Resumen
A través de esta investigación se pretende generar una serie de reflexiones acerca de elementos teóricos y prácticos que dificultan la aplicación del mercadeo estratégico en las Mipymes bogotanas y, que trae como consecuencia, la alta tasa de mortalidad de este tipo de organizaciones. Surgen entonces aspectos que van desde los mismos planteamientos teóricos y de aplicabilidad del mercadeo estratégico, (pues este último se concibe como una práctica afín a los recursos y dinámicas de la gran empresa, de allí que sea pertinente conocer cuáles son las barreras más frecuentes del mercadeo) hasta los elementos que permiten estimular y facilitar el uso del mercadeo estratégico en las Mipymes colombianas y latinoamericanas.
\end{abstract}

Se parte de la definición propuesta por la legislación colombiana de las Mipymes para pasar a recalcar su importancia dentro de la economía y plantear la problemática colombiana (a través de las cifras de su ciudad capital, Bogotá) de destrucción de riqueza por el cierre de empresas, en su mayoría Mipymes y cuya causalidad tiene significativos elementos de mala administración de mercadeo y comercial. Seguidamente se presentan los resultados logrados incluyendo los beneficios y las barreras encontradas en este contexto para la aplicación del mercadeo estratégico en las Mipymes. Se cierra el documento con una serie de consideraciones finales y recomendaciones.

\begin{abstract}
The purpose of this research is to generate a reflection about the theorist and practical elements that limit strategic marketing application in Bogota's SMEs which have as a consequence high mortality rate of this type of organizations. There are several aspects that rise from theoretical and applicable hypothesis within the strategic market because this practice is conceived as being related to the dynamics of big companies. This enables the understanding of the different barriers and of the elements that foster the use of strategic marketing within Colombian and Latin American SMEs.

We start from the Colombian law and its definition of SMEs in order to highlight their importance for the economy and to analyze the Colombian problematic (through figures of its capital, Bogotá) regarding the destruction of wealth due to company closures, especially SMEs, which lie especially on elements of bad marketing and commercial management. We also present the results achieved by including the benefits and barriers found within this context for the application of strategic marketing in SMEs. The article ends with a series of final considerations and recommendations.
\end{abstract}

\section{Palabras clave}

Mercadeo, estrategia de mercadeo, micro, pequeñas y medianas empresas

\section{Key Words}

Marketing, Marketing Strategy, Small and Medium Enterprises 
La importancia de las Mipymes en la economía, cada vez, se hace más evidente dentro de la generación de crecimiento económico en los países, debido tanto a su impacto directo sobre la generación de empleo como a su participación dentro del PIB de los mismos.

\section{INTRODUCCIÓN}

La definición de Mipymes en Colombia, según la legislación, categoriza a las empresas con base en el número de trabajadores o en sus activos totales en La Ley 905 de 2004 (Congreso de Colombia, 2004), que modificó a su vez La Ley Mipyme o Ley 590 de 2000 (Congreso de Colombia, 2000) fijando los parámetros siguientes:

Microempresa: aquella con planta de personal no superior a 10 trabajadores o activos totales, excluida la vivienda familiar, menores a 500 salarios mínimos mensuales legales vigentes (SMMLV) que para el 2010 correspondía aproximadamente a valores menores a US $\$ 130.000$. La Ley 905 considera que aquí básicamente estarían las fami-empresas y por eso excluye a la vivienda familiar del cálculo de los activos.

Pequeña empresa: planta de personal entre 11 y 50 trabajadores o activos totales entre 501 y menos de 5.000 SMMLV, lo cual implica aproximadamente activos entre US $\$ 130.000$ y US $\$ 1^{\prime} 300.000$.

Mediana empresa: planta de personal entre 51 y 200 trabajadores o activos totales entre 5.001 y 30.000 SMMLV, que corres-

\footnotetext{
Reseña de autor es

Sandra Patricia Rojas Berrio (Colombia)

Instituto Politécnico Nacional de México

srojasbe@poligran.edu.co

Administradora de Empresas y Magíster en

Doctorante en Ciencias Administrativas. Directora del

Departamento de Investigación Desarrollo e Innovación de la Fundación Politécnico Grancolombiano.

Ricardo Arturo Vega Rodríguez (Colombia)

Politécnico Grancolombiano

rvegarod@poli.edu.co

Químico Farmacéutico, Magíster en Administración. Profesor-Investigador del área de Mercadeo de la Fundación Politécnico Grancolombiano.
}

ponde a valores aproximadamente entre US\$1'300.000 y US\$7'800.000.

Gran empresa: más de 200 trabajadores o activos totales por más de 30.000 SMMLV, o sea, aproximadamente, más de US\$7'800.000.

De otro lado, la importancia de las Mipymes en la economía, cada vez, se hace más evidente dentro de la generación de crecimiento económico en los países, debido tanto a su impacto directo sobre la generación de empleo como a su participación dentro del PIB de los mismos. Para los países de rentas bajas representan un $31 \%$ de generación de empleo y un 15\% de su Producto Interno Bruto (PIB); para los países de rentas medias representan alrededor del 55\% de la generación de empleo y casi un $40 \%$ del PIB. Pero donde son más importantes económicamente es en el ámbito de los países de rentas altas donde logran cotas de alrededor del $65 \%$ en generación de empleo y representan un 50\% dentro de su PIB (The World Bank, 2003). De esta manera las Pymes se constituyen en un verdadero motor de desarrollo para cualquier tipo de sociedad.

Colombia no es ajena a esta tendencia mundial y así se puede observar como las Mipymes representan el 99,8\% de los negocios del país (Departamento Nacional de Planeación [DNP], 2006), un 67,2\% del empleo formal y un $38,7 \%$ de la generación del PIB (The World Bank, 2003). Para Bogotá, específicamente las Mipymes representan el 99\% de las empresas de la ciudad, que para 2005 eran 219.000 y que para el 2006 representaban el 27\% de las empresas constituidas en el país (Cámara de Comercio de Bogotá [CCB], 2006).

Dada la importancia que tienen las Mipymes en la economía colombiana, el 
Plan Nacional de Desarrollo 2006-2010 (PND) destina a este tipo de organizaciones un apartado titulado Estrategias específicas de productividad y competitividad para las microempresas y para las pymes (DNP, 2006). Dentro de las estrategias planteadas está la de Acceso a Servicios no Financieros de Desarrollo Empresarial (SDE) que busca potenciar las capacidades de los empresarios transfiriendo y desarrollando capacidades técnicas, tecnológicas y de gestión en las compañías.

Sin embargo, en cuanto a la problemática de constitución y liquidación de empresas en Bogotá está relacionado con el impacto de los programas de apoyo al desarrollo empresarial que, como se vio, son parte de la política pública, ya que a través de ella se refleja en la creación y liquidación de empresas, y es así como la Cámara de Comercio de Bogotá (CCB) destaca que en el trienio 2003-2005 se crearon en la capital del país 43.716 nuevas empresas, con un crecimiento del $16 \%$ respecto al trienio anterior (CCB, 2006). Pero en este mismo periodo se liquidaron 8.593 empresas (79\% microempresas, $16 \%$ pequeñas y $4 \%$ medianas empresas), que representaban 3,3 billones de pesos (alrededor de 1.704 millones de dólares) en capital acumulado (CCB, 2006), siendo casi el 20\% respecto de las empresas formadas. Es importante complementar estas cifras con las causas que generaron estos cierres (CCB, 2006):

1. Falta de veracidad de la información financiera y contable de las sociedades.

2. Exceso en el otorgamiento de préstamos a los socios.

3. Dificultades de las empresas para adaptarse a los cambios tecnológicos.

4. Baja utilización en la capacidad instalada o un alto índice de inventarios.
5. Baja participación de las exportaciones dentro del total de las ventas.

6. Incapacidad de formar un poder de marca y mantener la gestión comercial de sus productos.

7. Desequilibrio entre la estructura de costos y los precios.

8. Reducción del capital y aumento significativo de los pasivos.

9. Comportamiento negativo de sus ventas.

10. Dificultad en la gestión del apalancamiento financiero y del flujo de caja. (p.23)

Los puntos 5, 6 y 9 son una consecuencia directa de la gestión de mercadeo y comercial que desarrollan las empresas y hace pertinente el conocimiento de los aspectos que subyacen a esta faceta de la administración de las compañías y el papel que juega dentro de la labor gerencial a corto, mediano y largo plazo.

Teniendo en cuenta las anteriores consideraciones, se plantea la siguiente pregunta de investigación: ¿Cuál es la situación en las Mipymes bogotanas frente a la gestión de mercadeo que realizan? Por ende, el objetivo de investigación sería el estudiar la conceptualización y aplicación que hacen las Mipymes de Bogotá de las herramientas de gestión que propone el mercadeo estratégico.

Partiendo de éstos antecedentes, pregunta y objetivo de investigación, se plantean las siguientes hipótesis:

Aunque la planeación y el mercadeo estratégico son herramientas de gestión planteadas desde hace varias décadas, su difusión dentro de las Mipymes (Micro, Pequeñas y Medianas Empresas) de Bogotá, ha sido baja por las siguientes variables 
relativas a las características propias de las Mipymes de Bogotá:

1. Baja disponibilidad de recursos

2. Poca capacitación al interior de las organizaciones

3. Actitud negativa frente a estas herramientas, $y$

4. Dinámicas internas en el muy corto plazo, en este tipo de empresas.

Las anteriores variables son relativas a las características propias de las Mipymes de Bogotá. El apartado a continuación enunciará referentes teóricos que sustentan el desarrollo del trabajo de investigación.

\section{MERCADEO ESTRATÉGICO EN LAS MIPYMES}

Mullins, Walker, Boyd \& Larréché (2007) definen estrategia como "un modelo fundamental de metas presentes y planificadas, despliegue de recursos e interacciones de una organización con los mercados, los competidores y con otros factores ambientales" (p. 39). Refleja las respuestas a corto, mediano y largo plazo de las firmas a los desafíos y oportunidades planteados por el ambiente de negocios.

Se han efectuado variados desarrollos teóricos que plantean la implementación de la estrategia a través de un proceso formal de planeación estratégica y la cual Serna (2007) define como:

Un proceso mediante el cual quienes toman decisiones en una organización obtienen, procesan y analizan información pertinente, interna y externa, con el fin de evaluar la situación presente de la empresa, así como su nivel de competitividad con el propósito de anticipar y decidir sobre el direccionamiento de la institución hacia el futuro (p. 19).
Este direccionamiento estratégico se realiza definiendo los elementos centrales de la organización, como son la misión, visión, objetivos globales y ejes estratégicos, todo enmarcado dentro de los principios y valores corporativos (Serna, 2007).

Pero la planeación estratégica implica engranar una serie de elementos constitutivos de la organización y así, apelando a las cuatro perspectivas planteadas por Kaplan y Norton (1993), se deben generar y cohesionar las estrategias financieras, de procesos internos, de aprendizaje y crecimiento y las relacionadas con los clientes. Esta última perspectiva se cobija bajo la estrategia de mercadeo y constituye uno de los pilares fundamentales para el logro de los objetivos y el éxito organizacional.

Se define la estrategia de mercadeo como las "actividades de seleccionar y describir uno o más mercados meta, y de crear y mantener una mezcla de marketing que produzca intercambios mutuamente satisfactorios con los mercados meta" (Lamb, Hair \& McDaniel, 2006: 50). “El principal foco es el de identificar y crear una ventaja competitiva y se ve como una fuente de beneficios significativos para cualquier organización" (Murdoch, Blackey \& Blythe, 2001: 145), independientemente del tamaño de la misma. Podemos entonces afirmar que las compañías ejecutan sus estrategias para atraer consumidores y enfrentar de, una manera efectiva, múltiples elementos del entorno, tales como competidores, proveedores, agencias reguladoras, grupos de presión, recursos escasos, etc. (Knight, 2000) para que de esa manera se genere una mayor o menor orientación al mercado, y que se dará en la medida, que la empresa 
...esté focalizada en los procesos y actividades asociados con la consecución y satisfacción de los clientes, evaluando continuamente sus necesidades y deseos, y haciéndolo de una forma tal que haya un impacto medible y demostrable sobre el desempeño del negocio. (Palmer \& Pels, 2004: 60).

Esta orientación al mercado se convierte así, en un componente de la estrategia global de la organización que contribuirá positivamente a los resultados del negocio.

Un factor que también juega un papel importante dentro del análisis de las estrategias de mercadeo es la formalización que se haga de las mismas. Se debe diferenciar entre las estrategias formuladas y las implementadas (Palmer \& Pels, 2004), entre las estrategias explícitas y las implícitas, ya que unas y otras pueden jugar un rol significativo dentro de la gestión y el desempeño de las Mipymes sin implicar que la formalización necesariamente refleje el grado de orientación al mercado que tenga una compañía, aunque algunos estudios han mostrado que "las firmas con planes formales sobrepasan el desempeño de las que no los tienen" (Murdoch et al., 2001: 145).

La formalización se realiza bajo el plan estratégico de mercadeo, que como ya se comentó, tiene como "principal foco, la identificación y creación de una ventaja competitiva" (Murdoch et al., 2001: 145). Queda en este plan plasmada la escogencia de una posición relativamente atractiva para la firma, teniendo en cuenta la estructura de la industria donde se compite, las circunstancias propias de la organización y las posiciones de los competidores y articula todos los procesos y actividades en concordancia con la posición escogida (Porter, 1991).

Se subraya cómo la estrategia de mercadeo es importante para las Mipymes ya que provee un marco para los objetivos, decisiones y acciones y su esencia es la sensibilidad que tenga a los cambios y evoluciones del mercado (Knight, 2000). Pero a pesar de lo anterior los investigadores han detectado que la planeación estratégica de mercadeo no es tan ampliamente difundida en las Mipymes como pudiera pensarse (Lancaster \& Waddelow, 1998) y su aplicación se ve afectada primordialmente por tres elementos, interrelacionados entre sí, como son: 1) la actitud que se tiene frente al papel que debe jugar el mercadeo dentro de la firma, 2) el foco de atención que tienen los gerentes dentro de su labor en las Mipymes y 3) la disponibilidad de recursos de las mismas para implementar este proceso.

Respecto al elemento de la actitud frente al papel del mercadeo Murdoch et al. (2001) encontraron que en las Mipymes de Gales, el mercadeo es percibido como una actividad del corto plazo más que una función estratégica, lo cual lleva a una renuencia general a gastar dinero en mercadeo, pues éste se concibe como algo 'costoso' e inefectivo.

Por otro lado, Lancaster \& Waddelow (1998) detectaron como principales barreras los siguientes aspectos: "demasiado prolongado (generando un pobre retorno del esfuerzo invertido) y que además estará desactualizado en el momento en que se finaliza (desperdiciando preciosos recursos)" (p. 855); porque el mercadeo no se ve como un proceso continuo de adaptación al ambiente, sino como una tarea puntual que genera información inútil. No se percibe 
Lancaster \& Waddelow (1998) afirman que los directores de las Mipymes tienden a serpersonas cuyo foco de atención son los 'procesos', más que la estrategia. como una orientación o filosofía que debe guiar al negocio (Lancaster \& Waddelow).

Carson \& McCartan-Quinn (1995) añaden que "la actitud de las Mipymes frente a este proceso surge de un malentendido y es el de considerar que el mercadeo es imposible de cuantificar en términos de costo, y que sus resultados son intangibles y de difícil medición. Se considera más un costo que una inversión" (p.25). Así el mercadeo se convierte en una actividad periférica, ya que parece que no tuviera un impacto significativo sobre el desempeño del negocio (Carson \& McCartan-Quinn).

Respecto al foco de atención de los gerentes de las Mipymes en su trabajo, Murdoch et al. (2001) destacan que en este tipo de organizaciones sus gestores hacen más énfasis en el hacer que en el pensar. Se focalizan más en el día a día, que en la visión a mediano y largo plazo, la cual exige la planeación estratégica. Lancaster \& Waddelow (1998) afirman que los directores de las Mipymes tienden a ser personas cuyo foco de atención son los 'procesos', más que la estrategia. Muchos de ellos, por la propia estructura de las firmas que administran, desempeñan múltiples tareas asumiendo responsabilidades de producción, ventas, finanzas, contabilidad y recursos humanos. Esto hace que los directores de las Mypime tengan poco tiempo disponible para la planeación estratégica y realicen actividades de entrenamiento y desarrollo personal.

Añaden estos autores que pocos gerentes comprenden que pensar es una de sus más importantes actividades. Saltan de una tarea a otra y están orientados hacia el logro de resultados en el corto plazo. Su ética del trabajo y su cultura organizacional está basada en "lograr hacer las cosas".
Inclusive sienten que es mejor hacer las cosas, aunque puedan ser erróneas, a simplemente pensar acerca de ellas. Esto los lleva a planear soportados en la tradición de la firma y en las tácticas, ya que está es su realidad gerencial normal en el día a día. "La parte benéfica de esta situación es que los lleva a generar planes de acción que consideran pueden implementar" (Lancaster \& Waddelow, 1998: 856).

Lancaster \& Waddelow (1998) concluyen que este 'hacer' más que 'pensar' tiene cuatro consecuencias: 1) hay una constante presión y estrés con el trabajo, 2) se falla en la priorización y se convierten en solucionadores de problemas inmediatos, 3) se premia más la lealtad que la efectividad en sus empleados y 4) entran en un círculo vicioso del 'aquí y del ahora', creyendo que si no se resuelven las cuestiones urgentes no habrá futuro para la compañía. Como dice el adagio popular"'lo urgente no permite hacer lo importante”. De hecho este pensamiento se ha reforzado a lo largo de los años, ya que muchos de los gerentes de Mipymes raramente reciben una educación formal en gestión.

Y finalmente revisando el punto de los recursos disponibles en este tipo de firmas para desarrollar planeación estratégica Knight (2000, pp. 12-13) afirma que debido a su mismo tamaño las Mipymes carecen de "las capacidades, del poder de mercado, y de otros recursos con los que cuentan las grandes empresas”. Murdoch et al. (2001) aseveran que lo anterior se ve reflejado en la poca experiencia y entrenamiento en mercadeo dentro de este tipo de compañías.

No sólo se carece del conocimiento puntual sino que también, como se resaltó en el caso de los gerentes, no se dispone en gene- 
ral en las Mipymes del tiempo requerido para hacer planeación estratégica. Además, en la mayoría de los casos, este tipo de organizaciones no pueden ofrecer los paquetes salariales que dan las grandes compañías $y$ en muchas ocasiones tienen que conformarse con personal que ofrezca más lealtad y empatía que habilidades y aptitudes en su trabajo (Lancaster \& Waddelow, 1998).

Esta situación ha hecho que se hayan realizado un cierto número de intentos para producir teorías y modelos formales para la planeación estratégica en las Mipymes. A pesar de esto Murdoch et al. (2001) comen$\tan$ que

...ninguna de estas teorías y modelos han sido adoptados por las firmas y que no se han generado guías coherentes a este respecto. La evidencia encontrada muestra que la complejidad de la planeación estratégica de mercadeo es desalentadora para este tipo de organizaciones y genera prejuicios en contra de ella (p. 145).

Pero estos autores añaden que la cuestión se relaciona más con la forma en la cual los principios de mercadeo son aplicados que con la transferibilidad de los mismos a las Mipymes.

Lancaster y Waddelow (1998) llevan más lejos sus conclusiones afirmando que el proceso de planeación estratégica de mercadeo, tal como está planteada en la teoría moderna, es más apropiado para las grandes compañías porque se requiere una metodología operativamente más simple en el caso de las Mipymes. Pero reconocen que la planeación de mercadeo es como la contabilidad, hay principios comunes que pueden ser adapta- dos dependiendo de las necesidades específicas de cada organización. La problemática para estos autores radica primordialmente en encontrar una manera práctica de aplicar los principios de la planeación estratégica de mercadeo de una manera tal que contemple las características organizacionales propias de las Mipymes.

\section{METODOLOGÍA}

En respuesta a la pregunta que se ha planteado en este trabajo de investigación, se aplicó un diseño descriptivo transversal simple con Mipymes de la ciudad de Bogotá en el año $2008^{1}$.

Teniendo en cuenta la clasificación de Murdoch et al. (2001), se definieron áreas de investigación relacionadas con el conocimiento y actitud de las Mipymes respecto al mercadeo estratégico. Estas áreas fueron:

Tabla 1. Variables y preguntas en el instrumento de captura de información

\begin{tabular}{|l|c|}
\hline Variable & Pregunta (s) \\
\hline Caracterización demográfica & 1 a 9 \\
\hline Focalización en los clientes & 10 \\
\hline Ventaja competitiva & 11 \\
\hline Investigación de mercadeo & 12 \\
\hline Comunicaciones de mercadeo & 13 y 14 \\
\hline Formalización de los planes de mercadeo & 15 \\
\hline $\begin{array}{l}\text { Peso de cada horizonte temporal (corto, } \\
\text { mediano y largo plazo) dentro de la } \\
\text { planeación estratégica de mercadeo }\end{array}$ & 16 \\
\hline $\begin{array}{l}\text { Percepción de los beneficios de la } \\
\text { planeación de mercadeo }\end{array}$ & 17 \\
\hline $\begin{array}{l}\text { Barreras encontrada para realizar la } \\
\text { planeación estratégica de mercadeo }\end{array}$ & 18 \\
\hline
\end{tabular}

Fuente: Elaboración Propia

1. Listado de empresas encuestadas en Anexo 1

2. El cuestionario está disponible, contacte a los autores. 
Cabe notar, que la invitación a participar dentro del estudio se envió vía correo electrónico, a los integrantes de una base de datos compilada por los autores de esta investigación, que cubría Mipymes de Bogotá y en el cual se incluía un link al cuestionario ${ }^{2}$, para ser auto-aplicado. Como herramienta para el diligenciamiento y recolección de las encuestas, se usó la página española de Internet $w w w$. encuestafacil.com. Al finalizar la recolección de datos, se realizó el proceso de depuración y análisis en Excel,, y se hizo la estructuración de la información para hacer las gráficas y el análisis de resultados que se presentan a continuación:

\section{RESULTADOS}

Para realizar el proceso de análisis de información se adelantaron las siguientes etapas: Figura 1. Etapas del proceso de análisis de
información

\begin{tabular}{|c|c|}
\hline $\begin{array}{l}\text { Depuración de } \\
\text { información }\end{array}$ & \begin{tabular}{|l} 
Categorización de \\
las empresas
\end{tabular} \\
\hline $\begin{array}{l}\text { Análisis de datos } \\
\text { demográficos }\end{array}$ & $\begin{array}{l}\text { Análisis de la } \\
\text { gestión de mercadeo }\end{array}$ \\
\hline
\end{tabular}

Fuente: Elaboración Propia

A continuación se describen las acciones realizadas para cada una de las etapas del proceso de análisis de información:

La base de datos utilizada, a manera de marco de muestreo, contenía un total de 1074 registros iniciales que correspondieron a los correos electrónicos enviados. Se lograron 74 respuestas a la encuesta, lo que arrojó una tasa de respuesta bruta del 6,8\%; en el proceso de depuración se eliminaron 19, obteniendo así 55 respuestas válidas para el análisis, luego la tasa de respuesta neta fue del $5,1 \%$, cifra que se puede considerar adecuada y de acuerdo a las tasas de respuesta reportadas en estudios similares y que fluctúan entre 2,95\% y 10,19\% (Siu, Fan \& Lin, 2004).

Las razones para eliminar las 19 encuestas fueron: 14 que no fueron diligenciadas por completo, una fue realizada por una empresa que no estaba radicada en Bogotá, otra de una gran empresa (de acuerdo a los criterios usados para clasificarlas), una correspondía a un registro duplicado y dos casos tenían datos demográficos inconsistentes.

En cuanto a los datos demográficos, todos fueron diligenciados por los encuestados y reposan en los registros de la investigación. Las 55 empresas estaban localizadas en Bogotá y correspondían a la categoría de Mipymes.

Durante la siguiente etapa (Vega, 2010), se realizó una categorización con base en el número de empleados directos de la empresa tomando como referente la Ley 905 de 2004 y teniendo en cuenta que se trató de una información confiable suministrada por parte de las mismas empresas.

\section{Tabla 2. Número de empleados directos de} la organización

\begin{tabular}{|l|r|}
\hline ¿Cuántos empleados directos tiene la organización? \\
\hline 10 o menos (Microempresa) & 34 \\
\hline Entre 11 y 50 (Pequeña empresa) & 18 \\
\hline Entre 51 y 200 (Mediana empresa) & 3 \\
\hline Total & 55 \\
\hline
\end{tabular}

Fuente: Elaboración Propia

Se tiene un $62 \%$ de microempresas, 33\% de pequeñas empresas y un $5 \%$ de empresas medianas que contrasta bastante bien con los datos de empresas formadas en Bogotá en el trienio 2003-2005 que fueron en un 79\% 
microempresas, $16 \%$ pequeñas empresas, $4 \%$ de medianas empresas y un $1 \%$ de grandes empresas. Se puede afirmar que la muestra refleja de una manera adecuada la composición relativa de las Mipymes de Bogotá.

Para analizar datos demográficos como el sector industrial de la empresa, se tomó como referente el código CIIU (Clasificación Industrial Internacional Uniforme), se categorizaron por actividad industrial las empresas de la muestra y se obtuvieron los resultados presentados en la siguiente tabla.

Tabla 3. Sector industrial de las empresas

\begin{tabular}{|l|r|}
\hline ¿A qué sector industrial pertenece la empresa? \\
\hline Manufcatura & 15 \\
\hline Construcción & 1 \\
\hline Comerción & 6 \\
\hline Reparación y mantenimiento de autos & 1 \\
\hline Restaurantes & 1 \\
\hline Comunicaciones & 3 \\
\hline Actividades financieras & 1 \\
\hline Actividades inmobiliarias & 1 \\
\hline Prestación de servicios de salud & 7 \\
\hline Educación & 2 \\
\hline Otro & 17 \\
\hline Total & 55 \\
\hline
\end{tabular}

Fuente: Elaboración Propia

De los sectores identificados en esta pregunta se destacan manufactura con un $27 \%$, prestación de servicios de salud con un 13\%, comercio con un $11 \%$, y otros con un $31 \%$. Se tiene casi la tercera parte de la muestra compuesta por empresas manufactureras y dos terceras partes (incluyendo el 31\% de la clasificación otro) del sector servicios, lo que refleja el auge local, nacional e internacional que tiene este sector en las economías moder- nas y que es un promisorio sector de desarrollo para las Mipymes.

\section{Análisis de la gestión de mercadeo}

- Enfoque de gestión de mercadeo (Lamb et al., 2006; Mullins et al., 2007): se encontró que 39 de las 55 empresas tienen un enfoque de gestión de marketing y ventas centrado en la satisfacción y conocimiento del cliente complementado con un adecuado sistema de ventas y distribución, mientras que 15 compañías están divididas entre las orientaciones de producto ( 9 de las 55) y producción (6 de las 55), o sea más enfocadas en factores internos de la organización.

- Concepto de ventaja competitiva (Lamb et al., 2006; Porter, 1991): como era una respuesta de opción múltiple se presentaron 98 respuestas y solamente 7 empresas marcaron las tres características que definen la ventaja competitiva, a saber: 1) los beneficios aportados por el producto, 2) que el cliente los valore y 3 ) que sean diferenciales frente a la competencia.

Tabla 4. Formas de determinar la percepción y la actitud de los clientes frente al producto y sus competidores

\begin{tabular}{|l|r|}
\hline \multicolumn{2}{|l|}{ ¿Cómo determina en su empresa la percepción y actitud de sus clientes frente } \\
al producto y sus competidores? & 40 \\
\hline La conocen por su excelencia en el sector & 17 \\
\hline Encuestan a sus clientes & 29 \\
\hline Se detecta en el momento de la venta & 1 \\
\hline Contratana una empresa externa para que realice esta labor & 5 \\
\hline Otro & 92 \\
\hline Total &
\end{tabular}

Fuente: Elaboración Propia

- Proceso de detección de necesidades de los clientes (ver tabla 4): al ser una pregunta de opción múltiple, se recolectaron 92 respuestas, distribuyéndose de la siguiente manera: en 40 casos se anotó que este conocimiento lo logran a través de su experiencia en el sector, en 17 casos encuestan 
a los clientes, 29 realizan la determinación en el momento de la venta, en solo 1 caso contratan una empresa externa y en 5 apelan a otros elementos incluyendo una respuesta donde se reconoce que existe una gran deficiencia en este punto. Con base en las respuestas anteriores podemos afirmar que no existe mayoritariamente un proceso estructurado de investigación de mercado, ya que confían más en su conocimiento del sector y en la información que logren en los encuentros de venta con sus clientes (69 de las 92 respuestas). Solamente en 17 de las respuestas, se plasmo un interés estructurado de conocimiento de su mercado bien a través de encuestas propias (17) o bien contratando un tercero (en sólo 1 caso) que haga la labor.

- Elementos de la mezcla de comunicación (Lamb et al., 2006; Mullins et al., 2007): al ser una pregunta de opción múltiple, se obtuvieron 111 respuestas, es decir, prácticamente dos respuestas en promedio por cada una de las empresas encuestadas. En general los empresarios consideraron en mayor proporción la utilización de las relaciones públicas (29 de las 111), en proporciones similares el mercadeo directo (25 de las 111) y la fuerza de ventas (23 de las 111) y en menores proporciones la publicidad (19 de las 111) y las promociones de ventas (10 de las 111). Pero la finalidad de esta pregunta era inducir a la siguiente pregunta que realmente apunta a conocer la conceptualización de los empresarios sobre lo que ellos consideran es la mezcla de comunicación para un producto.

- Corroboración conceptual de los elementos de comunicación: la pregunta en referencia era si en la pregunta anterior seleccionó Publicidad, Relaciones Públicas y/o Mercadeo directo; ¿qué medios utiliza específicamente en cada caso? Se pretendía con esta pregunta conocer el nivel conceptual que los empresarios tienen de la mezcla de comunicación, la cual además es un concepto central de la estrategia de mercadeo. En la categoría: Publicidad, se obtuvieron 20 respuestas, para Relaciones Públicas 22 y para Mercadeo directo 16, es decir, que de las 111 respuestas obtenidas en la pregunta anterior solamente la mitad se comprometió con una respuesta abierta concreta. Es posible que lo anterior haya ocurrido dado que los encuestados no estaban seguros de sus respuestas y prefirieron dejarla en blanco, lo que se corrobora, en parte, con las respuestas dadas por los que sí diligenciaron la respuesta. Es así como de las 20 respuestas de publicidad, 11 correspondieron a la definición aceptada de Publicidad; de las 22 respuestas de Relaciones Públicas, 13 aparecieron correctas; y en el caso de Mercadeo directo 10 de los 16 contestaron correctamente (Lamb et al., 2006; Mullins et al., 2007). Lo anterior muestra un vacío conceptual en una proporción significativa de las empresas.

- Formalización de los planes de mercadeo: se presentan los resultados en la tabla siguiente.

\section{Tabla 5. Formalización de la estrategia en} planes de mercadeo

\begin{tabular}{|c|c|}
\hline \multicolumn{2}{|c|}{$\begin{array}{l}\text { ¿La estrategia de mercadeo de su organización se } \\
\text { formaliza en un plan de mercadeo escrito }\end{array}$} \\
\hline Sí & 26 \\
\hline No & 29 \\
\hline Total & 55 \\
\hline
\end{tabular}

Fuente: Elaboración Propia

- Un poco más de la mitad de los encuestados (29 de los 55) no formalizan sus planes de mercadeo, lo que muestra la significancia de las estrategias implicítas en este tipo de organizaciones.

- Horizonte temporal de planeación: prácticamente la tercera parte de los encuestados (19 de 55) hace planeación a corto plazo, casi la mitad (25 de 55) a mediano plazo y sólo 7 de los 55 la hacen a largo plazo, lo cual es coherente con las respuestas dadas a la pregunta anterior de la formalización de los planes. De las cuatro respuestas correspondientes a "Otro", tres contestaron que no tienen ningún elemento de planeación. Esta situación eleva la planeación a corto plazo 
a 22 de los 55 encuestados. O sea que en la muestra tenemos una baja formalización de la planeación y horizontes de tiempo cortos para visionar el futuro de la firma.
- Beneficios percibidos de la planeación de mercadeo: se verá la percepción de los empresarios a través de la siguiente tabla.

Tabla 6. Aspectos considerados como beneficiosos a partir de la planeación de mercadeo De los siguientes aspectos ¿cuál consideraría como beneficioso que se generan o pueden generar en su negocio a partir de la planeación de mercadeo en su organización?

\begin{tabular}{|l|r|}
\hline Impacta el crecimiento de los ingresos de la empresa & 43 \\
\hline Permite captar nuevos clientes & 48 \\
\hline Facilita la retención de los clientes actuales & 38 \\
\hline Ayuda en la definición de las características y componentes de los productos a ofrecer & 25 \\
\hline Aporta elementos para el análisis dela competencia & 22 \\
\hline No aporta ningún beneficio & 0 \\
\hline Otro & 2 \\
\hline Total & 178 \\
\hline
\end{tabular}

Fuente: Elaboración Propia

Al ser una pregunta de opción múltiple se obtuvieron 178 respuestas, en promedio tres por empresa $y$, como se observa en la gráfica, casi la mitad de las respuestas (86 de las 178) ponen de manifiesto al cliente como elemento central de la planeación tanto en su componente de captación (48 de las 178) como de retención de los consumidores (38 de las 178). Otra proporción importante de respuestas (43 de las 178) tiene relación con el crecimiento de la empresa, en 25 de las 178 respuestas se consideró que ayuda en la definición de las características del producto y en 22 de las 178 se registró que la planeación ayuda a realizar análisis de la competencia. La pregunta que surge es si estos beneficios están claros para los empresarios, ¿por qué la planeación estratégica de mercadeo se ejerce en una proporción relativamente baja? Parte de la respuesta surge de las barreras que se reportaron por los encuestados en la siguiente pregunta.

- Barreras encontradas a la planeación de mercadeo: por ser una pregunta de opción múltiple, se recolectaron 102 respuestas, las cuales se presentan en la siguiente tabla. 
Tabla 7. Barreras para la planeación de mercadeo De los siguientes elementos $i$, ¿cuáles considera que han sido barreras, al interior de su empresa, para la utilización e implementación de la planeación estratégica de mercadeo?

\begin{tabular}{|l|r|}
\hline Bajo entrenamiento en temas de planeación de mercadeo & 31 \\
\hline insuficiencia de recursos para fortalecer la implementación estratégica de mercadeo & 33 \\
\hline No aporta soluciones prácticas a los problemas comerciales de la organización & 4 \\
\hline Es una actividad que no genera un claro retorno de la inversión & 4 \\
\hline No se cuenta con suficiente tiempo para la planeación de mercadeo & 19 \\
\hline La planeación de mercadeo es un concepto poco práctico y es más un concepto teórico & 4 \\
\hline No ha encontrado ninguna barrera & 5 \\
\hline Otro & 2 \\
\hline Total & $\mathbf{1 0 2}$ \\
\hline
\end{tabular}

Fuente: Elaboración Propia

Las barreras que tienen que ver con problemas inherentes a la planeación en sí, tuvieron bajas proporciones dentro de las respuestas como son: a) no aporta soluciones prácticas a los problemas concretos de la compañía (en 4 de las 102 respuestas), b) es un concepto más teórico que práctico (en 4 de las 102 respuestas) y c) no genera un claro retorno a la inversión (en 4 de las 102).

Los elementos que surgen como barreras preponderantes son: a) la falta de recursos (en 33 de las 102 respuestas), b) de entrenamiento en el tema (en 31 de las 102) y c) de tiempo para desarrollar la planeación (en 19 de las 102).

Lo anterior es coherente con respuestas anteriores tales como la baja conceptualiza- ción de los elementos básicos de mercadeo, la baja formalización escrita de los planes de mercadeo y el reducido horizonte temporal donde se piensa estratégicamente aplicar.

Revisando la relación existente entre la búsqueda de información vía investigación de mercados, el horizonte de planeación y la elaboración de planes de mercadeo formales, se encuentra:

Que las organizaciones que no elaboran planes formales tienden a realizar sus procesos de planeación a menos de seis meses.

Que las organizaciones que han buscado información proveniente de investigaciones de mercado y hacen planes de mercadeo tienden a desarrollar horizontes de planeación de 6 a 18 meses. 
Tabla 8. Relación entre formalización de planes de mercadeo, investigación de mercados y el horizonte de planeación

\begin{tabular}{|c|c|c|c|c|c|c|}
\hline \multicolumn{7}{|c|}{$\begin{array}{c}\text { Tabla de contingencia. La planeación de mercadeo en su empresa, ¿a que horizonte de tiempo se realiza? } \\
\text { ¿Ha buscado alguna vez información proveniente de investigaciones de mercado? }\end{array}$} \\
\hline & & & & \multicolumn{3}{|c|}{$\begin{array}{l}\text { ¿Ha buscado alguna vez información } \\
\text { proveniente de investigaciones de mercado? }\end{array}$} \\
\hline & & & & SI & NO & TOTAL \\
\hline \multirow{9}{*}{$\begin{array}{l}\text { ¿La estrategia } \\
\text { de mercadeo } \\
\text { de su } \\
\text { organización } \\
\text { se formaliza } \\
\text { en un plan } \\
\text { de mercadeo } \\
\text { escrito? }\end{array}$} & \multirow{4}{*}{ SI } & \multirow{4}{*}{$\begin{array}{l}\text { La planeación } \\
\text { de mercadeo en } \\
\text { su empresa, ia } \\
\text { que horizonte } \\
\text { de tiempo se } \\
\text { realiza? }\end{array}$} & $\begin{array}{l}\text { Menos de } 6 \\
\text { meses }\end{array}$ & 2 & 1 & 3 \\
\hline & & & $\begin{array}{l}\text { De } 6 \text { a } 18 \\
\text { meses }\end{array}$ & 13 & 5 & 18 \\
\hline & & & $\begin{array}{l}\text { Más de } 18 \\
\text { meses }\end{array}$ & 2 & 2 & 4 \\
\hline & & & Total & 17 & 8 & 25 \\
\hline & \multirow{5}{*}{ NO } & \multirow{5}{*}{$\begin{array}{l}\text { La planeación } \\
\text { de mercadeo en } \\
\text { su empresa, ¿a } \\
\text { que horizonte } \\
\text { de tiempo se } \\
\text { realiza? }\end{array}$} & $\begin{array}{l}\text { Menos de } 6 \\
\text { meses }\end{array}$ & 4 & 12 & 16 \\
\hline & & & $\begin{array}{c}\text { De } 6 \text { a } 18 \\
\text { meses }\end{array}$ & 2 & 3 & 5 \\
\hline & & & $\begin{array}{c}\text { Más de } 18 \\
\text { meses }\end{array}$ & 2 & 1 & 3 \\
\hline & & & Ninguno & 3 & 1 & 4 \\
\hline & & & Total & 11 & 17 & 28 \\
\hline
\end{tabular}

Fuente: Elaboración Propia

\section{DISCUSIÓN Y RECOMENDACIONES}

Un primer elemento que surge del estudio es el hecho de que los empresarios encuestados tienen una clara conceptualización del "deber ser” estratégico de mercadeo en las organizaciones, pues expresan que el cliente tiene que ser el centro de gestión de la empresa y reconocen que el mercadeo ocupa un lugar preponderante dentro de la gestión de la compañía. Pero esta conceptualización del papel del mercadeo en la gestión y el logro de los objetivos, no se complementa con una aplicación de estos conceptos en el día a día de la organización.

Una de las causas que se encontraron para la poca aplicación del mercadeo estratégico en los empresarios es el bajo nivel de conocimiento que tienen respecto a conceptos básicos de mercadeo, como son el de ventaja competitiva y mezcla de comunicación, que a su vez son elementos centrales a definir dentro de los planes estratégicos de la Mipyme. Si no hay un conocimiento del tema, difícilmente se puede esperar que los empresarios incorporen dichos aspectos dentro de la gestión comercial de sus empresas.

Ese conocimiento y actitud frente a la planeación estratégica de mercadeo, también se ven reflejados en dos elementos formales del proceso como son: 1) la baja generación de planes de mercadeo escritos y 2) el corto horizonte de tiempo en el cual están llevando a cabo sus procesos de planeación de mercadeo. De igual manera, existe una proporción importante de empresarios que llevan sus procesos de gestión de mercadeo de una manera informal, pensando en el impacto a corto plazo y descuidando la visión de futuro que deberían generar para la organización. Ésta situación es similar a la que se encontró en estudios realizados en otros contextos.

La barrera más frecuente que dificulta la planeación y la gestión estratégica de mercadeo tiene relación con el bajo entrenamiento de los empleados en temas de planeación de 
A su vez, este estudio abre

las puertas

para que otras

investigaciones

revisen el

enfoque que

tienen los

programas

de fomento al

emprendimiento

y a las

Mipymes que

se desarrollan

en el medio

colombiano,

especialmente,

en cuanto a los

componentes

que incorporan

la gestión de

mercadeo, para

así evaluar

sidichos

programas, sí

proveen a los

empresarios de

los conceptos

teóricos $y$ de las

herramientas

prácticas que

les permitan

realizar una

gestión de

mercadeo

efectivay

útil para el

desempeño de las

empresas. mercadeo, lo que confirma y pone en claro que los mismos empresarios reconocen las carencias de conocimiento que poseen en este tema. Otras barreras significativas son la carencia de recursos y de tiempo para implementar el proceso.

En la parte final de la encuesta, se encontró que existe una actitud positiva frente a los beneficios que traería el plantear y planear estrategias de mercadeo, pero esta actitud no está complementada con los conceptos prácticos que se requieren, ya que en el proceso de usar esta herramienta de gestión las Mipymes se encuentran con barreras provenientes de vacíos conceptuales y de recursos que les impiden convertir esa actitud positiva, en elementos concretos de la gestión empresarial.

Aunque el presente estudio es descriptivo y transversal, y se realizó tomando una muestra pequeña de encuestados, los elementos detectados sobre el enfoque de gestión de mercadeo, en las Mipymes bogotanas, son de gran valor einterés, tanto que se pretende continuar con esta línea de investigación.

A su vez, este estudio abre las puertas para que otras investigaciones revisen el enfoque que tienen los programas de fomento al emprendimiento y a las Mipymes que se desarrollan en el medio colombiano, especialmente, en cuanto a los componentes que incorporan la gestión de mercadeo, para así evaluar si dichos programas, sí proveen a los empresarios de los conceptos teóricos y de las herramientas prácticas que les permitan realizar una gestión de mercadeo efectiva y útil para el desempeño de las empresas.

Este estudio también llama la atención con respecto a los elementos de seguimiento que se le hace a la gestión a largo plazo de mercadeo y permite conocer si están más enfocados en otro tipo de elementos, como por ejemplo los aspectos eminentemente financieros, que en los de planeación.

Algunas de las limitaciones del presente trabajo de investigación tienen que ver con el siguiente aspecto: 1) en la revisión realizada, se encontraron una serie de barreras que impiden la aplicación del mercadeo estratégico en las Mipymes en diferentes contextos, por ello es importante que se repliquen este tipo de esfuerzos investigativos para lograr comprender cuáles de las mismas, se presentan en los países latinoamericanos y cuáles son propias de las particularidades organizacionales y del entorno de las Mipymes en cada uno de los países del área.

Por otra parte, se deben generar trabajos conjuntos entre Academia - Gobierno - Gremios de Mipymes para que se logren sinergias tanto a nivel de conocimiento de las dinámicas de las Mipymes regionales como la concepción y aplicación de políticas de fomento a este sector de la economía. La anterior propuesta es también una necesidad ya vista en otros autores colombianos (Montoya, 2010).

\section{Bibliografía}

1. Cámara de Comercio de Bogotá. (2006, noviembre). Dinámica de la inversión empresarial en Bogotá 2003-2005. Extraído el 1 de diciembre de 2008, de http://camara.ccb.org.co/ documentos/636_2007_5_3_10_0_13_Inversion_Empresarial_2003_-_2005.pdf.

2. Carson, D. \& McCartan-Quinn, D. (1995). Non-practice of Theoretically Based Marketing in Small Business-Issues Arising and their Implications [Versión electrónica]. Journal of Marketing Theory \& Practice, vol.3(4), pp.24-32. 
3. Colombia, Departamento Nacional de Planeación. (2006). Plan Nacional de Desarrollo 2006-2010. Extraído el 1 de diciembre de 2008 de http://www.dnp.gov.co/PortalWeb/ Portals/0/archivos/documentos/GCRP/PND_2006_2010/Capi_4_FINAL.pdf.

4. Congreso de Colombia. (2000, 10 de julio). Ley 590 de 2000 (Número de Diario Oficial - 44.078). Bogotá, D.C.: Imprenta Nacional de Colombia. Disponible en: http://www. imprenta.gov.co/.

5. Congreso de Colombia. (2004, 2 de agosto). Ley 905 de 2004 (Número de Diario Oficial - 45.628). Bogotá, D.C.: Imprenta Nacional de Colombia. Disponible en: http://www. imprenta.gov.co/.

6. Hernández, R., Fernández, C. \& Baptista, P. (1998). Metodología de la investigación (2a. ed.). México: McGraw-Hill Interamericana.

7. Kaplan, R. \& Norton, D. (1993). Putting the Balanced Scorecard to Work [Versión electrónica]. Harvard Business Review, vol.71(5), pp.134-147.

8. Knight, G. (2000). Entrepreneurship and Marketing Strategy: The SME Under Globalization [Versión electrónica]. Journal of International Marketing, vol.8(2), pp.12-32.

9. Lancaster, G. \& Waddelow, I. (1998). An Empirical Investigation into the Process of Strategic Marketing Planning in SMEs: Its Attendant Problems, and Proposals Towards a New Practical Paradigm [Versión electrónica]. Journal of Marketing Management, vol.14, pp.853-878.

10. Lamb, C., Hair, J. \& McDaniel, C. (2006). Marketing (8a. ed.). México: Thomson.

11. Montoya, A. (en prensa). Situación de la competitividad de las Pymes en Colombia: elementos actuales y retos. Agronomía Colombiana.

12. Mullins, J., Walker, O., Boyd, H. \& Larréché, J. (2007). Administración de marketing: Un enfoque en la toma estratégica de decisiones (5a. ed.). México: McGraw-Hill Interamericana.

13. Murdoch, H., Blackey, H. \& Blythe, J. (2001). Beliefs and attitudes of Welsh SMEs to marketing [Versión electrónica]. Journal of Targeting, Measurement and Analysis for Marketing, vol.10(2), pp.143-155.

14. Palmer, R. \& Pels, J. (2004). Marketing Practice and Market Orientation: An Exploratory International Study [Versión electrónica]. Journal of Euromarketing, vol.14(1/2), pp.59-86.

15. Porter, M. (1991). Towards a Dynamic Theory of Strategy [Versión electrónica]. Strategic Management Journal, vol.12, pp.95-117.

16. Serna, H. (2003). Gerencia estratégica (9a ed.). Bogotá: 3R Editores.

17. Siu, W., Fan, W. \& Lin, T. (2004). Strategic marketing practices and the performance of Chinese small and medium-sized enterprises (SMEs) in Taiwan [Versión electrónica]. Entrepreneurship \& Regional Development, vol.16(2), pp.161-178.

18. Vega, R. (2009). Conocimiento, percepción y aplicación del mercadeo estratégico en Mipymes (Micro, pequeñas y medianas empresas) de Bogotá. Manuscrito enviado para publicación.

19. The World Bank. (2003). Small and Medium Enterprises across the Globe: A New Database (Policy research working paper 3127). Washington: Ayyagari, M., Beck, T. \& DemirgüçKunt, A. Extraído el 3 de diciembre de 2008, de http://www-wds.worldbank.org/external/ default/WDSContentServer/WDSP/IB/2003/09/30/000094946_0309160409277/ Rendered/PDF/multi0page.pdf. 